\title{
Bis(3)-Tacrine Inhibits the Sustained Potassium Current in Cultured Rat Hippocampal Neurons
}

\author{
R.-J. WEN ${ }^{*}$, D. HUANG ${ }^{1^{*}}$, Y. ZHANG ${ }^{1}$, Y.-W. LIU ${ }^{2}$ \\ * R.-J.W. and D.H. contributed equally to this work as first authors.
}

${ }^{1}$ Department of Physiology, School of Medicine, Jianghan University, Wuhan, People's Republic of China, ${ }^{2}$ Department of Anatomy, School of Medicine, Jianghan University, Wuhan, People's Republic of China

Received March 23, 2016

Accepted November 21, 2016

On-line February 28, 2017

\section{Summary}

Bis(3)-tacrine is a dimeric AChE inhibitor derived from tacrine with a potential to treat Alzheimer's disease. It was recently been reported to act as a fast off-rate antagonist of NMDA receptors with moderate affinity. In the present study, we aimed to explore whether bis(3)-tacrine could modulate the function of native sustained potassium current in cultured rat hippocampal neurons using whole-cell patch-clamp technique. We found that bis(3)-tacrine inhibited the amplitude of sustained potassium current in a reversible and concentration-dependent manner, with a potency two orders of magnitude higher than that of tacrine. The inhibition was voltage-independent between 0 to $+60 \mathrm{mV}$. The $\mathrm{IC}_{50}$ values for bis(3)-tacrine and tacrine inhibition of sustained potassium current were $0.45 \pm 0.07$ and $50.5 \pm 4.8 \mu \mathrm{M}$, respectively. $I-V$ curves showed a more potent inhibition of sustained potassium current by bis(3)-tacrine $(1 \mu \mathrm{M})$ compared to tacrine at the same concentration. Bis(3)-tacrine hyperpolarized the activation curve of the current by $11.2 \mathrm{mV}$, albeit leaving the steady-state inactivation of the current unaffected.

\section{Key words}

Bis(3)-tacrine - Sustained potassium current • Hippocampus • Alzheimer's disease

\section{Corresponding author}

Y.-W. Liu, Department of Anatomy, School of Medicine, Jianghan University, Wuhan 430056, People's Republic of China. Fax: +86-27-84226723. E-mail: liu-yuwei@163.com

\section{Introduction}

$\operatorname{Bis}(3)$-tacrine, (1,3- $N$-heptylene-bis-9,9'-amino1,2,3,4-tetrahydroacridine), a novel dimeric acetylcholineesterase (AChE) inhibitor derived from tacrine, has been demonstrated to inhibit $\mathrm{AChE}$ and $\gamma$-aminobutyric acid subtype $\mathrm{A}\left(\mathrm{GABA}_{\mathrm{A}}\right)$ receptors (Carlier et al. 1999, Li et al. 2007), and it was recently reported to act as uncompetitive $N$-methyl-D-aspartate (NMDA) receptor antagonist with fast off-rate (UFO) and moderate affinity, which interacts with its targets only during pathological states with no physiological activation (Luo et al. 2010). It can reverse cognitive impairment resulted from scopolamine in both water maze and object recognition tasks (Han et al. 2012), might represent a promising drug candidate for various neurodegenerative disorders, including Alzheimer's disease (AD). To date, no data about its actions on potassium currents in central nervous system neurons have been reported.

This work aimed to explore whether bis(3)-tacrine was able to modulate voltage-gated potassium channels as reported by many authors for the modulation of rat hippocampal neurons by tacrine (Wooltorton and Mathie 1993), huperzine A ( $\mathrm{Li}$ and $\mathrm{Hu}$ 2002a, $\mathrm{Li}$ and $\mathrm{Hu} 2002 \mathrm{~b}$ ); of DRG neurons and Kv1.2 encoded potassium channels expressed in oocytes by bis(7)-tacrine (Nie et al. 2007). In the present study, using whole-cell patch-clamp recording, we aimed at investigating the effects of bis(3)-tacrine (compared to 
tacrine) on sustained potassium current in cultured rat hippocampal neurons.

\section{Methods}

\section{Cell culture}

Primary cultures of hippocampal neurons from Sprague-Dawley rats at embryonic day 18 were prepared as previously described with modifications (Liu et al. 2008a, Liu et al. 2008b). In brief, hippocampi were dissected and incubated with $0.25 \%$ trypsin for $15 \mathrm{~min}$ at $37^{\circ} \mathrm{C}$, and then mechanically dissociated using a fire-polished Pasteur pipette. The resulting cell suspension was diluted at a density of $1 \times 10^{5}$ cells $/ \mathrm{ml}^{-1}$ with high glucose Dulbecco's modified Eagle's medium solution containing $10 \%$ fetal bovine serum, and plated in 35-mm dishes coated with poly-L-lysine $\left(20 \mu \mathrm{g} / \mathrm{ml}^{-1}\right)$. Cells were incubated at $37^{\circ} \mathrm{C}$ in a humidified incubator with $5 \% \mathrm{CO}_{2}$. After approximately $24 \mathrm{~h}$, the medium was replaced with a serum-free Neurobasal medium containing $\mathrm{B}_{27}$ supplement and $0.5 \mathrm{mM}$ L-glutamine to inhibit the growth of glia cells. This medium was subsequently given half-changes twice weekly, and neurons were cultured for 7-14 days before use in experiments. All animal experimental procedures were reviewed and approved by the Animal Care and Use Committee at Jianghan University, and were performed in accordance with the National Institutes of Health guidelines on animal care.

\section{Whole-cell electrophysiological recordings}

Whole-cell patch-clamp recordings were performed at room temperature $\left(22-25^{\circ} \mathrm{C}\right)$ using an Axon 200B amplifier (Molecular Devices Co., Union City, CA, USA), a Digidata 1320A A/D converter (Molecular Devices Co.) and pCLAMP 10.2 software (Molecular Devices Co.). Current traces were low-pass filtered at $1-5 \mathrm{kHz}$ and sampled at frequencies of 5-20 kHz. Series resistance was compensated by $75-85 \%$. Linear leak and residual capacitance currents were subtracted online using a $\mathrm{P} / 6$ protocol. The liquid junction potential measured to be less than $3 \mathrm{mV}$ was not compensated. The membrane potential was held at $-50 \mathrm{mV}$, unless noted otherwise. Neurons were placed in an extracellular medium containing (in $\mathrm{mM}$ ): $135 \mathrm{NaCl}, 5 \mathrm{KCl}, 1 \mathrm{CaCl}_{2}$, $2 \mathrm{MgCl}_{2}, 10$ glucose, 10 HEPES and 0.001 tetrodotoxin ( $\mathrm{pH}$ was adjusted to 7.3 with $\mathrm{NaOH}$, and the osmolality was adjusted to $\sim 340 \mathrm{mosmol} / \mathrm{kg}$ with sucrose). The patch-pipettes were filled with an intracellular solution containing (in $\mathrm{mM}$ ): 125 potassium gluconate, $20 \mathrm{KCl}$, $2 \mathrm{MgCl}_{2}, 1 \mathrm{CaCl}_{2}, 10$ EGTA, 10 HEPES, and $5 \mathrm{MgATP}$ with $\mathrm{pH} 7.3$ and $\sim 315 \mathrm{mosmol} / \mathrm{kg}$ in osmolality. Drug solutions were prepared in extracellular medium and applied to neurons by using a rapid solution exchange system (SF-77B Perfusion Fast-Step, Warner Instruments, Hamden, CT). With this system, the $10-90 \%$ rise time of the junction potential at an open pipette tip was $<2 \mathrm{~ms}$ (Liu et al. 2008a, Liu et al. 2008b). Neurons were bathed constantly in extracellular medium flowing from one barrel between drug applications.

\section{Chemicals and applications}

All drugs and chemicals used were purchased from Sigma-Aldrich Chemical Company (St Louis, MO, USA), except Alkylene-linked tacrine dimer bis(3)-tacrine $\cdot 2 \mathrm{HCl}$, that was kindly provided by Prof. Yifan Han (The Hong Kong Polytechnic University, Hong Kong, China). Unless indicated, all media and supplements used for cell culture were purchased from Gibco (Carlsbad, CA, USA).

\section{Data analysis}

Average values are expressed as mean \pm S.E.M, with $n$ equal to the number of cells studied. Statistical significance of results was assessed using Student's $t$-test or analysis of variance (ANOVA), as noted. Statistical analysis of concentration-response data was performed using the nonlinear curve-fitting program ALLFIT (DeLean et al. 1978), which uses an ANOVA procedure. Values reported for concentration-response analysis are those obtained by fitting the data to the equation:

$$
\mathrm{Y}=E_{\max } /\left[1+\left(\mathrm{EC}_{50} / \mathrm{X}\right)^{n}\right]
$$

where $\mathrm{X}$ and $\mathrm{Y}$ are concentration and response, respectively, $E_{\max }$ is the maximal response, $\mathrm{EC}_{50}$ is the concentration yielding 50 percent of maximal effect ( $\mathrm{EC}_{50}$ for activation, $\mathrm{IC}_{50}$ for inhibition), and $n$ is the slope factor.

The steady-state activation/inactivation curves were fitted with the Boltzmann equation:

$$
G / G_{\max }=1 /\left\{1+\exp \left[-\left(V-V_{1 / 2}\right) / k\right]\right\}
$$

where $G / G_{\max }$ is the normalized conductance, $V$ is membrane potential, $V_{1 / 2}$ is the potential for half-maximal activation or inactivation, and $k$ is the slope factor. Mono- or bi-exponential functions were used to fit current decay and recovery from inactivation data. 


\section{Results}

\section{Bis(3)-tacrine inhibited the sustained potassium current}

The sustained potassium current was eliminated by $20 \mathrm{mM}$ tetraethylammonium (TEA) $(n=5$, data not shown). In the present study we found that the sustained potassium current could also be reversibly inhibited by bis(3)-tacrine (Fig. 1A), in a concentration-dependent manner $\left(10^{-9}-10^{-4} \mathrm{M}\right)$ (Fig. 1B). In contrast, the inhibition by tacrine of sustained potassium current is similar, but the potency is about two orders of magnitude below that of bis(3)-tacrine ( $\mathrm{IC}_{50}$ values of $50.5 \pm 4.8 \mu \mathrm{M}$ compared to $0.45 \pm 0.07 \mu \mathrm{M}, p<0.05, n=9$, Student's $t$ test).
A

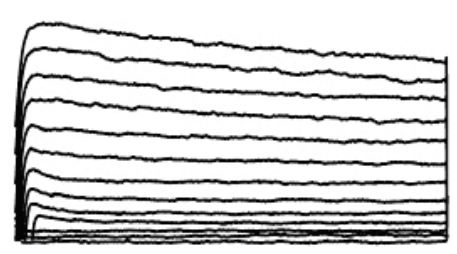

Control

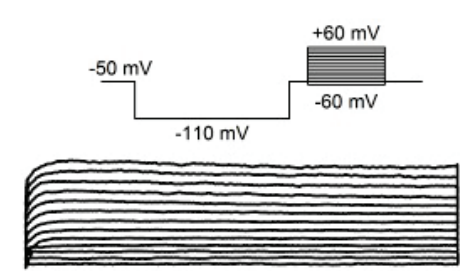

Bis(3)-tacrine
B

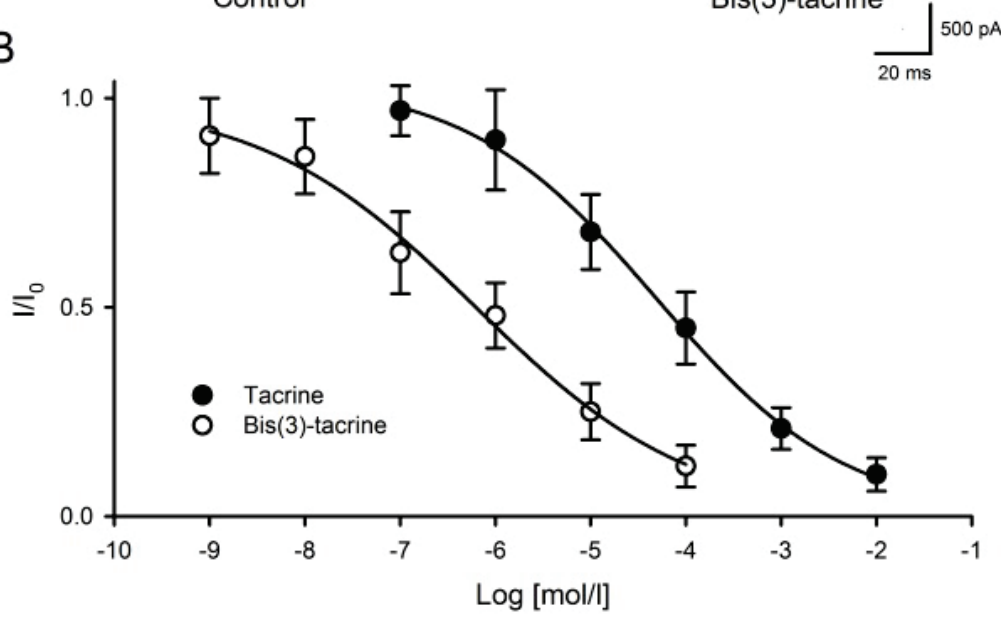

Fig. 1. Bis(3)-tacrine inhibited the sustained potassium current. (A) Family of currents evoked in control conditions and with $10^{-7} \mathrm{M}$ bis(3)-tacrine by depolarizing command pulses from $-60 \mathrm{mV}$ to $+60 \mathrm{mV}(10 \mathrm{mV}$ steps) following a $400 \mathrm{~ms}$ hyperpolarizing prepulse at $-110 \mathrm{mV}$ with a $50 \mathrm{~ms}$ interval at $-50 \mathrm{mV}$ to inactivate fast transient potassium current. (B) The concentrationresponse curves of bis(3)-tacrine $(n=9)$ and tacrine $(n=9)$
Effect of membrane potential on inhibition of sustained potassium current

As illustrated in Figure 2A, bis(3)-tacrine/tacrine suppressed the sustained potassium current. They both depressed the I-V curves relative to control at all different holding potentials. At the depolarizing voltage of $+60 \mathrm{mV}$, the amplitude of sustained potassium current was reduced by $16.8 \%$ and $52.6 \%$ compared to control values by tacrine $(1 \mu \mathrm{M})$ and bis(3)-tacrine $(1 \mu \mathrm{M})$, respectively.

To explore whether the change in percentage current in the presence of drugs is voltage-dependent or not, the bar chart graph in Figure 2B, was drawn by plotting of percentage current $\left(\mathrm{I} / \mathrm{I}_{0}\right)$ (ordinate) against the membrane potential (abscissa). It is evident that in the presence of bis(3)-tacrine $(1 \mu \mathrm{M})$, the change in percentage current is independent of membrane potential from 0 to $+60 \mathrm{mV}$ (ANOVA, $p>0.05 ; n=5$ ).
A

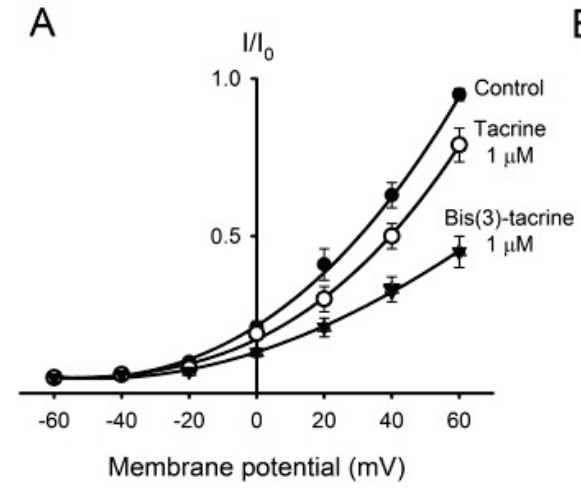

B

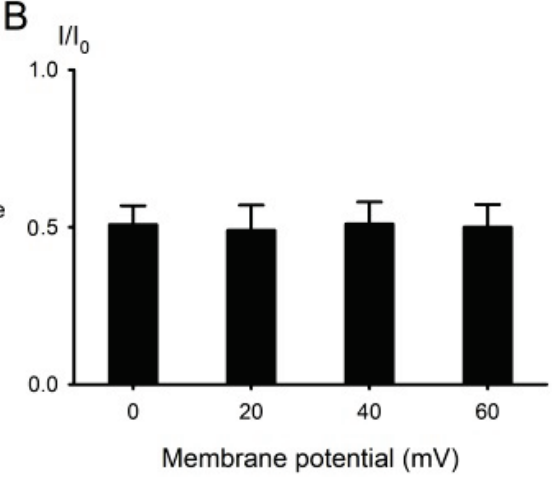

Fig. 2. Effect of membrane potential on inhibition of sustained potassium current by bis(3)-tacrine/tacrine. (A) Current/ voltage $(I-\bigvee)$ curves showed a more potent inhibition of sustained potassium current by bis(3)-tacrine $(1 \mu \mathrm{M})$ compared to tacrine at the same concentration. (B) The percentage current $\left(\mathrm{I} / \mathrm{I}_{0}\right)$ at $0,+20,+40$ and $+60 \mathrm{mV}$ in the presence of bis(3)-tacrine $(1 \mu \mathrm{M})(n=5, p>0.05$ versus $0 \mathrm{mV}$ ). 
Effect of bis(3)-tacrine on the steady-state activation and inactivation of sustained potassium current

We further examined the effect of bis(3)-tacrine on the steady-state activation of sustained potassium current. The conductance-voltage $(G-V)$ curves in Figure 3A show a leftward shift in the presence of bis(3)-tacrine $(1 \mu \mathrm{M})$. As a result, the steady-state activation curve became steeper. The half-maximal activation voltage was shifted to the hyperpolarizing direction by $11.2 \mathrm{mV}$ (from $-7.8 \pm 1.1$ to $-19.0 \pm 3.2 \mathrm{mV}$ ) $(n=6)$ by bis(3)-tacrine.
A

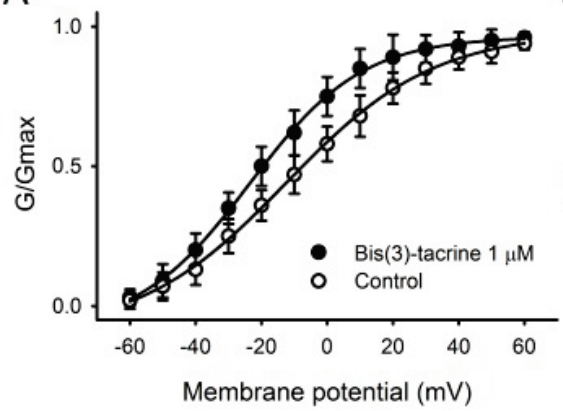

B

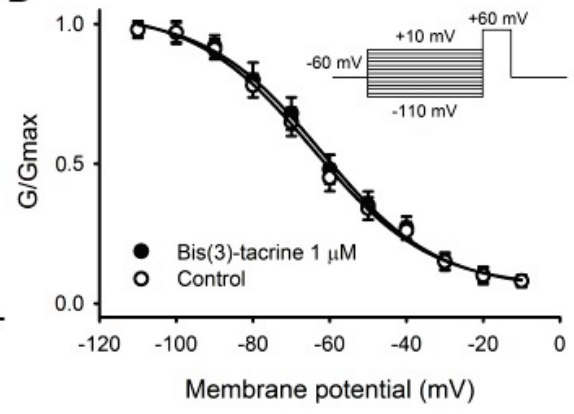

Fig. 3. Effect of bis(3)-tacrine on the steady-state activation (A) and inactivation (B) of sustained potassium current. Pulse protocols similar to those in Figure 1 were used to study the steady-state activation. Steady-state inactivation was studied using pulse protocols with hyperpolarizing prepulses from -110 to $10 \mathrm{mV}$ (10 mV steps) followed by a step to a fixed voltage $(+60 \mathrm{mV})$.
To measure the steady-state inactivation, depolarizing steps to $+60 \mathrm{mV}$ for $500 \mathrm{~ms}$ were elicited after a series of 2,000 ms prepulses from -110 to $+10 \mathrm{mV}$ in $10 \mathrm{mV}$ increments. The $G-V$ curves in Figure 3B show that bis(3)-tacrine had no effect on the inactivation of sustained potassium current. The voltage values at halfmaximal inactivation conductance for sustained potassium current in the absence and presence of bis(3)-tacrine $(1 \mu \mathrm{M})$ are $-60.9 \pm 5.2$ and $-59.5 \pm 5.0 \mathrm{mV}$ (Student's $t$ test, $p>0.05 ; n=7$ ), respectively.

\section{Discussion}

Sustained potassium current is one of the main voltage-activated potassium currents, and plays a critical role in maintaining neuronal excitability (Pongs 1999). In the present study, it is the first time that we report bis(3)-tacrine can inhibit sustained potassium current in cultured rat hippocampal pyramidal neurons.

Our results show that the inhibition by bis(3)-tacrine and tacrine are both concentrationdependent, the extent of inhibition by bis(3)-tacrine of sustained potassium current shows a potency two orders of magnitude higher compared to tacrine (Fig. 1B). This has also been noted for other AChE inhibitors, such as huperzine $\mathrm{A}$ ( $\mathrm{Li}$ and $\mathrm{Hu} 2002 \mathrm{a}, \mathrm{Li}$ and $\mathrm{Hu} 2002 \mathrm{~b}$ ), galantamine (Pan et al. 2003b), rivastigmine (Pan et al. 2003a) and bis(7)-tacrine (Nie et al. 2007, Li et al. 2010) suppress potassium currents in both central and peripheral neurons.

Furthermore, we analyzed the possible mechanisms underlying these inhibitions. It had been reported that both cholinesterase inhibitors, huperzine $\mathrm{A}$ and bis(7)-tacrine, inhibited sustained potassium current in voltage-dependent and voltageindependent way, respectively ( $\mathrm{Li}$ and $\mathrm{Hu}$ 2002a, $\mathrm{Li}$ and $\mathrm{Hu} 2002 \mathrm{~b}$, Nie et al. 2007). It can be seen from our result shown in Figure 2B that there was no significant change in percentage current $\left(\mathrm{I} / \mathrm{I}_{0}\right)$ during the alteration of membrane potentials in the presence of bis(3)-tacrine as compared with control, that means the inhibition of sustained potassium current by bis(3)-tacrine is voltage-independent. Therefore, it is unlikely for bis(3)-tacrine to inhibit the currents by blocking the pore of potassium channel.

The kinetic properties of sustained potassium current were also significantly affected by bis(3)-tacrine. It was interesting that in the presence of bis(3)-tacrine the activation curve of sustained potassium current shifted leftwards. Earlier activation may indicate an increase in current amplitude; however, a decrease in sustained potassium current was clearly observed in the present study. One possible explanation could be that although the activation curve was slightly shifted by about $11 \mathrm{mV}$, bis(3)-tacrine is binding with its acting sites in the potassium channel in the whole process, letting less current leak through, thus decreasing the amplitude of the potassium current. Besides, bis(3)-tacrine did not significantly affect the inactivation curve of sustained potassium current. These phenomena coincided with those of inhibition of sustained potassium current by huperzine A in hippocampal neurons ( $\mathrm{Li}$ and $\mathrm{Hu} 2002 \mathrm{~b}$ ); 
but in some degree differ from those of inhibition by bis(7)-tacrine in DRG neurons and Kv 1.2 encoded potassium channels expressed in oocytes ( $\mathrm{Nie}$ et al. 2007), which shows that bis(7)-tacrine shifts the activation/inactivation curves of sustained potassium current leftwards in both preparations, the effect being more pronounced for the latter. The difference was unlikely induced by using different species of neurons coming from central and peripheral nervous system, respectively, but probably suggest the different degree of protonized structures among these three compounds may lead to their binding to different depths of acting sites in the potassium channels.

It is generally accepted that the massive neuronal death which occurs in $\mathrm{AD}$ is due to apoptosis (Zhu et al. 2006). Growing evidence has shown that in the process of neuronal apoptosis and neurodegeneration induced by up-regulating potassium channel activities, neuronal potassium homeostasis is seriously disrupted ( $\mathrm{Yu}$ 2003). The finding that TEA, and other potassium channel blockers attenuate cell apoptosis in cultured cortical neurons (Yu et al. 1997, Yu et al. 1999, Wang et al. 2000) support this speculation. Many AChE inhibitors are able to inhibit potassium currents, such as tacrine (Rogawski 1987, Kraliz and Singh 1997), huperzine $\mathrm{A}$ ( $\mathrm{Li}$ and $\mathrm{Hu}$ 2002a, $\mathrm{Li}$ and $\mathrm{Hu}$ 2002b), donepezil (Zhang et al. 2004), galantamine (Pan et al. 2003b), bis(7)-tacrine (Nie et al. 2007, Li et al. 2010), etc. One possible pharmacological implication of our finding is that the blockade of voltage-activated potassium current by bis(3)-tacrine might lead to suppression of apoptosis and a substantial increase in cell survival.
Bis(3)-tacrine has been demonstrated to inhibit $\mathrm{AChE}$ and $\gamma$-aminobutyric acid subtype $\mathrm{A}\left(\mathrm{GABA}_{\mathrm{A}}\right)$ receptors (Carlier et al. 1999, Li et al. 2007). Recently, Luo et al. (2010) reported that bis-(propyl)-cognitin, which is the same compound as bis(3)-tacrine in our report, is an uncompetitive $N$-methyl-D-aspartate (NMDA) receptor antagonist with fast off-rate (UFO) and may represent a promising drug candidate for various neurodegenerative disorders. Further studies need to answer the question that if bis(3)-tacrine influence GABA-activated current, as we studied before (Zhou et al. 2009). Han et al. (2012) reported that it can reverse cognitive impairment resulted from scopolamine in both water maze and object recognition tasks; and under the same condition, the relative potency to improve cognitive capacity was 5-20 folds over that of tacrine. This compound could be referred as a multi-target, potential effective drug for treatment of $\mathrm{AD}$ and thus may be favourable for suppressing the over-activation of potassium channels that emerges in the pathogenesis of $\mathrm{AD}$.

\section{Conflict of Interest}

There is no conflict of interest.

\section{Acknowledgements}

This work was supported by the Foundation of Science and Technology Bureau of Wuhan, China (201250499145-27 to Y.-W. Liu), the Foundation of Hubei Provincial Department of Education (B2016286 to R.-J. WEN) and Jianghan University startup funds to R.-J. WEN, the National Natural Science Foundation of China (81273846 to Y. ZHANG).

\section{References}

CARLIER PR, HAN YF, CHOW ES, LI CP, WANG H, LIEU TX, WONG HS, PANG YP: Evaluation of short-tether bis-THA AChE inhibitors. A further test of the dual binding site hypothesis. Bioorg Med Chem 7: 351-357, 1999.

DELEAN A, MUNSON PJ, RODBARD D: Simultaneous analysis of families of sigmoidal curves: application to bioassay, radioligand assay, and physiological dose-response curves. Am J Physiol 235: E97-E102, 1978.

HAN RW, ZHANG RS, CHANG M, PENG YL, WANG P, HU SQ, CHOI CL, YIN M, WANG R, HAN YF: Reversal of scopolamine-induced spatial and recognition memory deficits in mice by novel multifunctional dimers biscognitins. Brain Res 1470: 59-68, 2012.

KRALIZ D, SINGH S: Selective blockade of the delayed rectifier potassium current by tacrine in Drosophila. J Neurobiol 32: 1-10, 1997.

LI C, CARLIER PR, REN H, KAN KK, HUI K, WANG H, LI W, LI Z, XIONG K, CLEMENT EC, XUE H, LIU X, LI M, PANG Y, HAN Y: Alkylene tether-length dependent gamma-aminobutyric acid type A receptor competitive antagonism by tacrine dimers. Neuropharmacology 52: 436-443, 2007. 
LI XY, ZHANG J, DAI JP, LIU XM, LI ZW: Actions of bis(7)-tacrine and tacrine on transient potassium current in rat DRG neurons and potassium current mediated by $\mathrm{K}(\mathrm{V}) 4.2$ expressed in Xenopus oocyte. Brain Res 1318: 23-32, 2010.

LI Y, HU GY: Huperzine A, a nootropic agent, inhibits fast transient potassium current in rat dissociated hippocampal neurons. Neuroscience Lett 324: 25-28, 2002a.

LI Y, HU GY: Huperzine A inhibits the sustained potassium current in rat dissociated hippocampal neurons. Neuroscience Lett 329: 153-156, $2002 \mathrm{~b}$.

LIU YW, LUO JL, REN H, PEOPLES RW, AI YX, LIU LJ, PANG YP, LI ZW, HAN YF, LI CY: Inhibition of NMDA-gated ion channels by bis(7)-tacrine: whole-cell and single-channel studies. Neuropharmacology 54: 1086-1094, 2008a.

LIU YW, LI CY, LUO JL, LI WM, FU HJ, LAO YZ, LIU LJ, PANG YP, CHANG DC, LI ZW, PEOPLES RW, AI YX, HAN YF: Bis(7)-tacrine prevents glutamate-induced excitotoxicity more potently than memantine by selectively inhibiting NMDA receptors. Biochem Biophys Res Commun 369: 1007-1011, $2008 \mathrm{~b}$.

LUO J, LI W, ZHAO Y, FU H, MA DL, TANG J, LI C, PEOPLES RW, LI F, WANG Q, HUANG P, XIA J, PANG Y, HAN Y: Pathologically activated neuroprotection via uncompetitive blockade of N-methyl-D-aspartate receptors with fast off-rate by novel multifunctional dimer bis(propyl)-cognitin. J Biol Chem 285: 19947-19958, 2010.

NIE H, YU WJ, LI XY, YUAN CH, PANG YP, LI CY, HAN YF, LI ZW: Inhibition by bis(7)-tacrine of native delayed rectifier and KV1.2 encoded potassium channels. Neurosci Lett 412: 108-113, 2007.

PAN Y, XU X, WANG X: Rivastigmine blocks voltage-activated K+ currents in dissociated rat hippocampal neurons. Br J Pharmacol 140: 907-912, 2003a.

PAN YP, XU XH, WANG XL: Galantamine blocks delayed rectifier, but not transient outward potassium current in rat dissociated hippocampal pyramidal neurons. Neurosci Lett 336: 37-40, 2003 b.

PONGS O: Voltage-gated potassium channels: from hyperexcitability to excitement. FEBS Lett 452: 31-35, 1999.

ROGAWSKI MA: Tetrahydroaminoacridine blocks voltage-dependent ion channels in hippocampal neurons. Eur J Pharmacol 142: 169-172, 1987.

WANG X, XIAO AY, ICHINOSE T, YU SP: Effects of tetraethylammonium analogs on apoptosis and membrane currents in cultured cortical neurons. J Pharmacol Exp Ther 295: 524-530, 2000.

WOOLTORTON JR, MATHIE A: Block of potassium currents in rat isolated sympathetic neurones by tricyclic antidepressants and structurally related compounds. Br J Pharmacol 110: 1126-1132, 1993.

YU SP: Regulation and critical role of potassium homeostasis in apoptosis. Prog Neurobiol 70: 363-386, 2003.

YU SP, YEH C, STRASSER U, TIAN M, CHOI DW: NMDA receptor-mediated K+ efflux and neuronal apoptosis. Science 284: 336-339, 1999.

YU SP, YEH CH, SENSI SL, GWAG BJ, CANZONIERO LM, FARHANGRAZI ZS, YING HS, TIAN M, DUGAN LL, CHOI DW: Mediation of neuronal apoptosis by enhancement of outward potassium current. Science 278: 114-117, 1997.

ZHANG W, JIN HW, WANG XL: Effects of presenilins and beta-amyloid precursor protein on delayed rectifier potassium channels in cultured rat hippocampal neurons. Acta Pharmacol Sin 25: 181-185, 2004.

ZHOU L, LIU YW, PEOPLES RW, YANG M, TIAN X, AI YX, PANG YP, LI ZW, HAN YF, LI CY: Mechanism of bis(7)-tacrine inhibition of GABA-activated current in the cultured rat hippocampal neurons. Neuropharmacology 57: 33-40, 2009.

ZHU X, RAINA AK, PERRY G, SMITH MA: Apoptosis in Alzheimer disease: a mathematical improbability. Curr Alzheimer Res 3: 393-396, 2006. 